\title{
Surface acoustic wave generation and detection using graphene interdigitated transducers on lithium niobate
}

\author{
A. S. Mayorov, N. Hunter, W. Muchenje, C. D. Wood, M. Rosamond, E. H. Linfield, \\ A. G. Davies, and J. E. Cunningham \\ School of Electronic and Electrical Engineering, University of Leeds, Leeds LS2 9JT, United Kingdom
}

(Received 13 December 2013; accepted 6 February 2014; published online 25 February 2014)

\begin{abstract}
We demonstrate the feasibility of using graphene as a conductive electrode for the generation and detection of surface acoustic waves at $100 \mathrm{~s}$ of $\mathrm{MHz}$ on a lithium niobate substrate. The graphene interdigitated transducers (IDTs) show sensitivity to doping and temperature, and the characteristics of the IDTs are discussed in the context of a lossy transmission line model. (C) 2014 AIP Publishing LLC. [http://dx.doi.org/10.1063/1.4866273]
\end{abstract}

Interdigitated transducers (IDTs) are commonly used for the generation and detection of surface acoustic waves (SAWs) on piezoelectric substrates including GaAs, quartz, and lithium niobate $\left(\mathrm{LiNbO}_{3}\right) . \quad \mathrm{LiNbO}_{3}$ has a high electro-mechanical coupling coefficient, and is selected for many sensors and high-frequency signal processing applications using SAWs. ${ }^{1}$ For most cases, it is desirable to use inert metals (such as Au or Pt) for IDTs, as they are not affected by ambient conditions. However, such dense metals cannot be used at high frequencies owing to mass-loading effects, whereby the inertia of the transducer fingers reduces the magnitude of the generated acoustic wave; the dense metal will also reflect the mechanical waves. To mitigate these deleterious effects, high frequency IDTs are usually made from metals with a relatively low density such as $\mathrm{Al}$, and are often embedded into the substrate to reduce undesirable reflections. ${ }^{2}$ Another approach has been used at room temperature is to form IDTs from a two-dimensional electron system (2DES) in an AlGaN/GaN heterostructure, but this has a significant drawback since the insertion loss is greater than $80 \mathrm{~dB}$, mainly due to the resistive loss arising from the 2DES. ${ }^{3}$

Graphene is a promising candidate for high frequency IDTs since it is conductive, whilst also being the thinnest and lightest material that can be easily processed by conventional photolithography. ${ }^{4}$ As such, no significant mass loading is expected from IDTs made of graphene. In this paper, the feasibility of using IDTs made from graphene for SAW generation and detection is demonstrated. We show SAW operation at a frequency of $164 \mathrm{MHz}$ on a $\mathrm{LiNbO}_{3}$ substrate both at room and at low temperatures, and the effect of graphene doping on the transmitted signals.

The geometry of the device and a micrograph are presented in Figure 1. The IDTs were designed as shown in Figure 1 with a $24 \mu \mathrm{m}$ periodicity and an acoustic aperture of $64 \mu \mathrm{m}$. Each IDT is composed of 16 pairs of electrodes and the separation between the two IDTs was $294 \mu \mathrm{m}$. CVD graphene (supplied by Graphene Laboratories Inc.) was transferred onto a $128^{\circ}$ Y-rotated one-side polished single-crystal black $\mathrm{LiNbO}_{3}$ substrate. Optical lithography using a bi-layer resist lift-off process was then used to fabricate metallic contacts to the graphene. First, a layer of LOR-3A photoresist was spun at $4000 \mathrm{rpm}$ for $40 \mathrm{~s}$ and baked at $145^{\circ} \mathrm{C}$ for $60 \mathrm{~s}$; the LOR-3A was baked at this relatively low temperature to avoid cracking the $\mathrm{LiNbO}_{3}$ owing to the pyroelectric nature of the substrate. A layer of Shipley S1813 photoresist was then spun at $4000 \mathrm{rpm}$ for $40 \mathrm{~s}$ and baked at $115^{\circ} \mathrm{C}(60 \mathrm{~s})$. After UV exposure $\left(100 \mathrm{~mW} / \mathrm{cm}^{2}\right)$, and development for $100 \mathrm{~s}$ in $\mathrm{MF} 319, \mathrm{Cr} / \mathrm{Au}(5 \mathrm{~nm} / 50 \mathrm{~nm})$ ohmic contacts were deposited onto the graphene using an electron-beam evaporator, and the pattern lifted-off in Shipley MF319. Finally, a single layer of Shipley S1813 was patterned to form the graphene IDT pattern by subsequent etching using an Oxygen plasma-asher (4 millibars, $50 \mathrm{~W}, 120 \mathrm{~s}$ ). Graphene is transparent on a $\mathrm{LiNbO}_{3}$ substrate, with no apparent contrast. Therefore, an optical micrograph was taken before the resist layer was finally removed to show the resulting geometry of the device (Figure 1(a)). All measurements were performed on devices with the resist removed by immersion in acetone and subsequent cleaning in isopropyl alcohol and water, unless otherwise stated.

To investigate the quality of graphene transferred onto $\mathrm{LiNbO}_{3}$, a Raman spectrum was measured using a HORIBA Jobin Yvon Raman spectrometer with a $633 \mathrm{~nm}$ HeNe laser and $5 \mathrm{~mW}$ power of circular polarised light (Figure 2(a)). Noting that the substrate is highly anisotropic, the graphene signal has to be determined by subtraction of a background signal measured for the same orientation of the substrate. The spectrum shows the common features related to single layer graphene with a small D-peak, caused by point-like defects. ${ }^{5,6}$ For resistance measurements, a Hall bar of $3 \mu \mathrm{m}$ width and $40 \mu \mathrm{m}$ length was fabricated and characterised at $2 \mathrm{~K}$ using standard low-frequency $(83 \mathrm{~Hz})$ lock-in techniques (Figure 2(b)). The low temperature $(2 \mathrm{~K})$ carrier concentration found from Shubnikov-de Haas oscillations and Hall effect measurements was $\sim 2.4 \times 10^{12} \mathrm{~cm}^{-2}$, while the mobility was found to be $3000 \mathrm{~cm}^{2} \mathrm{~V}^{-1} \mathrm{~s}^{-1}$. We expect that the quality of graphene used in transport measurements is very similar to the graphene used for IDT fabrication, since the source of graphene and all subsequent processing were the same.

The fabricated graphene IDTs had a periodicity of $\lambda=24 \mu \mathrm{m}$, with both the width and the spacing of fingers equal to $6 \mu \mathrm{m}$, and were aligned to the $\mathrm{X}$ direction of the substrate. ${ }^{7}$ Assuming no mass loading effects and perfect crystallographic orientation to the $\mathrm{X}$ direction, this period corresponds to a central frequency of $166 \mathrm{MHz}$, since $f_{0}=v_{0} / \lambda$, where $v_{0}$ is the SAW phase velocity $\left(3992 \mathrm{~ms}^{-1}\right.$ on $\left.\mathrm{LiNbO}_{3}\right){ }^{8}$ No $(<1 \mathrm{nA})$ DC leakage current was detected between the two sides of each IDT array when biased at $1 \mathrm{~V}$, 
(a)

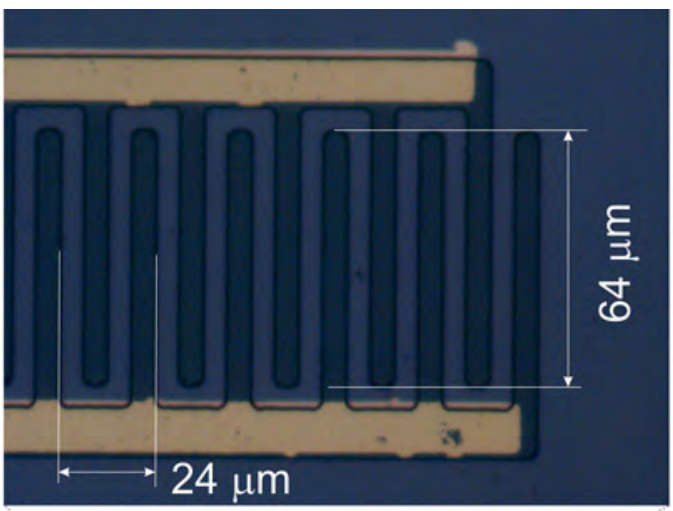

(b)

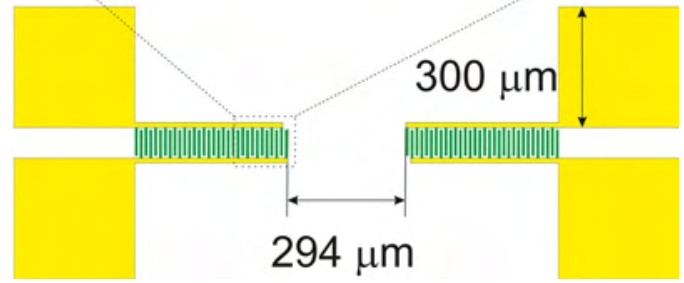

FIG. 1. Geometry of the device. (a) Optical image of a graphene IDT covered by $1.3-\mu \mathrm{m}$-thick $\mathrm{S} 1813$ photoresist. The interdigital periodicity is $24 \mu \mathrm{m}$ and the acoustic aperture is $64 \mu \mathrm{m}$. (b) Graphene IDT configuration showing the chromium-gold bond pads and busbars (yellow) and 16 pairs of graphene IDT fingers (green). The separation between IDTs is $294 \mu \mathrm{m}$.

indicating that the etching processes effectively isolated adjacent fingers. Frequency domain characterisation of the bonded device was then performed using a network analyzer (Agilent E8364B). Typical transmission $\left(\mathrm{S}_{21}\right)$ and reflection $\left(\mathrm{S}_{11}, \mathrm{~S}_{22}\right)$ curves measured at room temperature are shown in Figure 3. The black solid line in Figure 3(a) with $-45 \mathrm{~dB}$ maximum transmission corresponds to the frequency characteristic of an as-deposited pair of graphene IDTs. The resistance of the graphene should affect the efficiency of SAW generation and detection by the IDTs. ${ }^{9}$ To investigate this, we doped the graphene by exposing it to nitric acid vapour for 5 and 20 min. ${ }^{10}$ The sample was placed near a beaker with $10 \mathrm{ml}$ of $60 \%$ nitric acid and covered by a $400 \mathrm{ml}$ beaker. The transmission curves are shown in Figure 3(a) (red dotted line and blue dashed line, respectively). The best transmission (of $-35 \mathrm{~dB}$ ) was found after 20 min interaction between the graphene and nitric acid vapour, although we note that the characteristics were found to revert back to the original traces over a period of $\sim 24 \mathrm{~h}$ after 5 min doping, owing to evaporation of the dopant (green solid line in Figure 3(a)). This instability in the dopant level after nitric acid exposure is typical of such doping, and is presumably caused by gradual desorption. ${ }^{11}$

Figure 3(b) shows both the transmission and reflection curves after $20 \mathrm{~min}$ of doping. The similarity between reflection losses in the IDTs located at either end of the substrate (comparing the dotted and dashed lines in Figure 3(b)) indicates good uniformity in the deposited graphene, and the absence of any large macroscopic defects or breaks in the film.

At the higher doping levels, additional small oscillations (shown by the red arrow) of the transmission central peak appear (Figure 3(b), black curve) with an amplitude modulation of $\sim 0.5 \mathrm{~dB}$. We attribute these oscillations to the
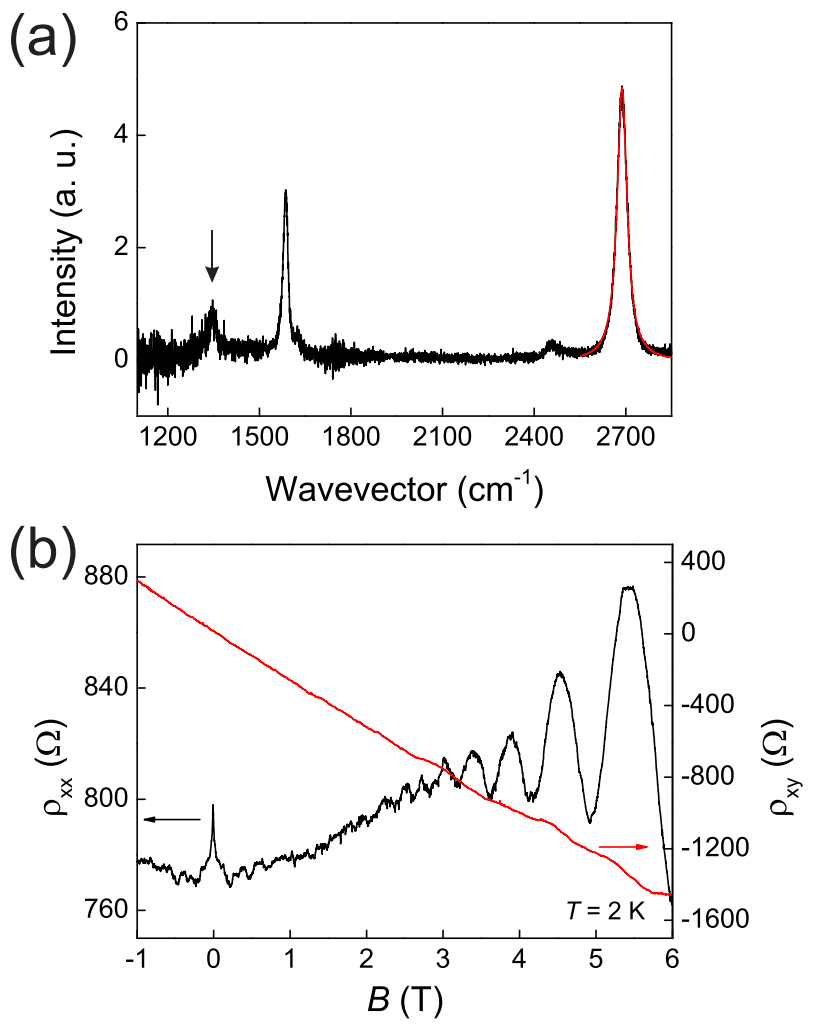

FIG. 2. Graphene characterisation. (a) Raman spectrum of graphene IDTs, after removal of background signal from the lithium niobate. ${ }^{7}$ The black arrow indicates the D-peak. The 2D-peak is at $2688 \mathrm{~cm}^{-1}$, and the G-peak is at $1586 \mathrm{~cm}^{-1}$. The red curve is a Lorentzian fit to the 2D-peak, indicating that the sample is single-layer graphene. ${ }^{6}$ (b) Diagonal (black) and Hall (red) resistivities for graphene on $\mathrm{LiNbO}_{3}$ as a function of magnetic field measured at $2 \mathrm{~K}$.

reflection of the SAW from the boundary of the substrate placed on a distance of $1.2 \mathrm{~mm}$ from the centre of an IDT (since a reflection from the opposite IDT would give a modulation of $0.27 \mathrm{~dB}$, based on the measured $-36 \mathrm{~dB}$ insertion loss). ${ }^{12}$

Low temperature SAW measurements of the asdeposited graphene are shown in Figure 4(a), highlighting the weak temperature dependence of the transmission; the

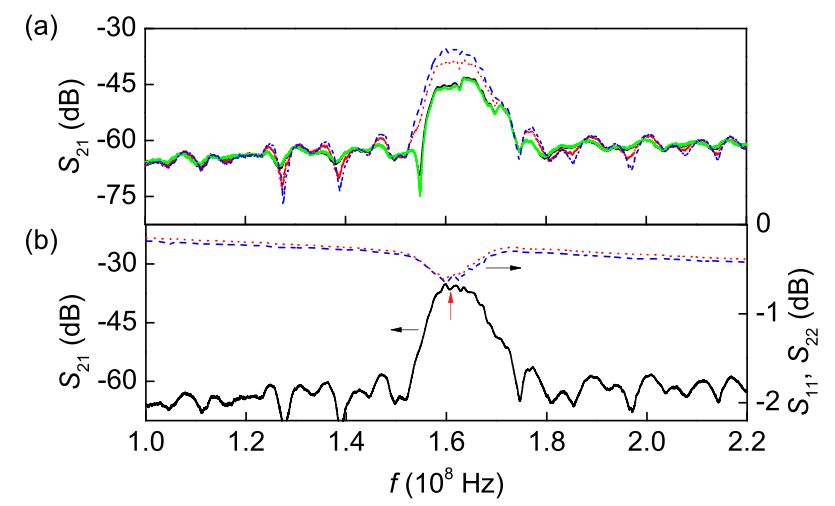

FIG. 3. S-parameters measured for doped graphene IDTs at room temperature as a function of frequency. (a) Transmission $\left(S_{21}\right)$ measured for an as-deposited graphene IDT (black solid line). Red dotted and blue dashed lines correspond to $5 \mathrm{~min}$ and $20 \mathrm{~min}$ doping in nitric acid vapour, respectively. The green solid line is measured $24 \mathrm{~h}$ after doping by nitric acid vapour for 5 minutes. (b) Transmission $\left(S_{21}\right.$, ) and reflection $\left(S_{22}\right)$ for graphene after exposure to nitric acid vapour for $20 \mathrm{~min}$ shown as the blue dashed line in (a). 

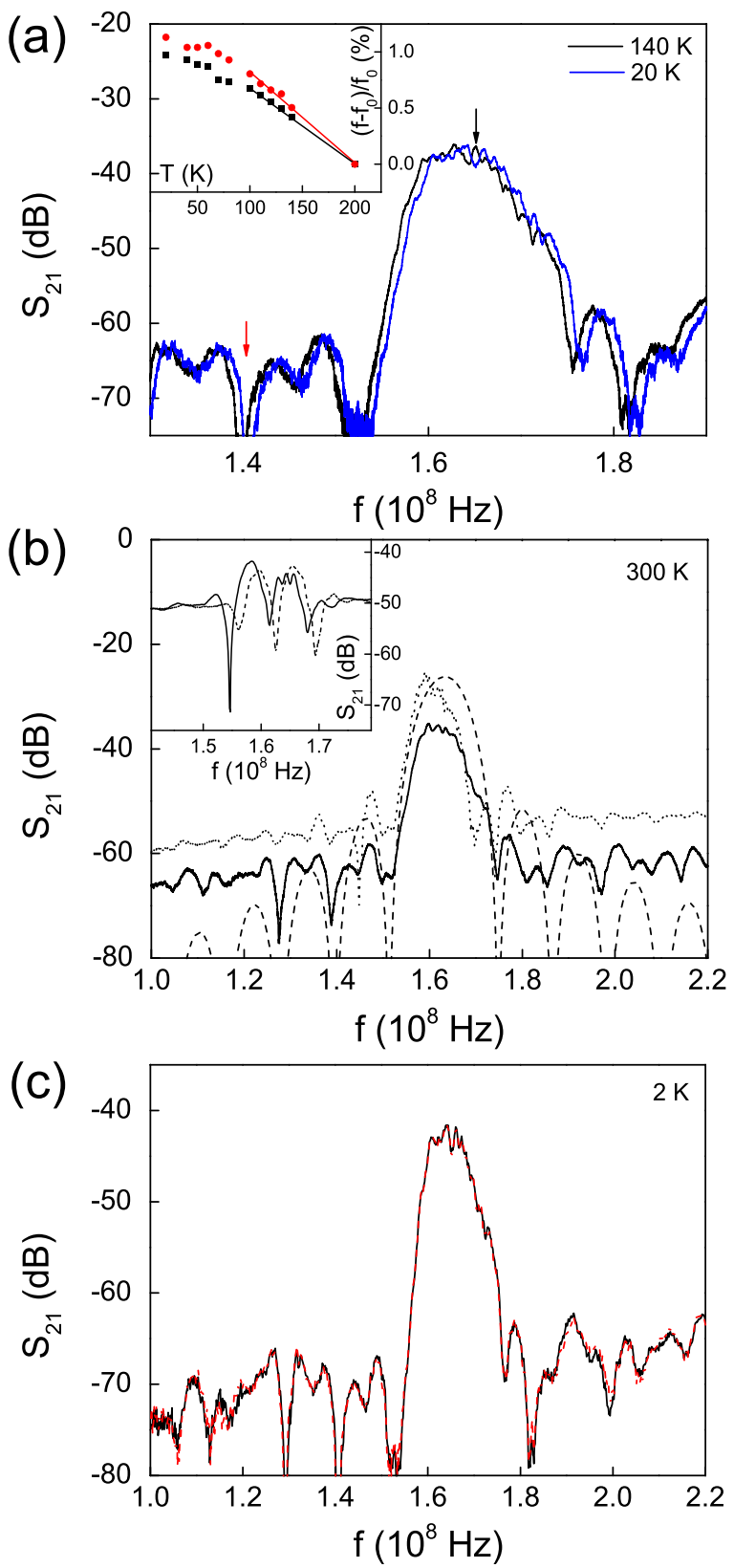

FIG. 4. (a) Transmission as a function of frequency for two temperatures. Inset: the temperature dependence of the shift of the maximum (black dots) and minimum (red dots) values of $S_{21}$ with respect to the positions at $200 \mathrm{~K}$ indicated by the black and red arrows, respectively, expressed as a percentage of the value at $200 \mathrm{~K}$; the black and red solid lines are best fits for the black squares and red circles. The calculated temperature coefficient of velocity is

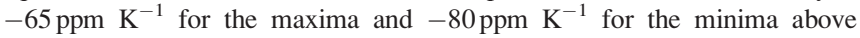
$100 \mathrm{~K}$. (b) Transmission as a function of frequency measured at room temperature for graphene electrodes (solid curve, from Figure 3(b)), $\mathrm{Cr} / \mathrm{Au}$ electrodes (dotted line), and calculated using COM theory (dashed curve) for metal electrodes of zero thickness and the geometry presented in Figure 1. Inset: Transmission of a second device before (solid line) and after (dashed line) resist removal. (c) Transmission as a function of frequency for zero magnetic field (black solid curve) and 6 $\mathrm{T}$ (red dashed curve), measured at $2 \mathrm{~K}$.

peak response was found to shift towards higher frequencies with decreasing temperature. The temperature coefficient of the central frequency shift is between $-65 \pm 6 \mathrm{ppm} / \mathrm{K}$ and $-80 \pm 6 \mathrm{ppm} / \mathrm{K}$ above $100 \mathrm{~K}$, given by the slope of the relative shift for the minimum and maximum (see Figure 4(a), inset), in agreement with previous experiments performed with metal SAW transducers on $\mathrm{LiNbO}_{3}$ at room temperature $(-72 \mathrm{ppm} / \mathrm{K}){ }^{8}$
The resistance of an individual finger pair, $R_{g}=16 \mathrm{k} \Omega$, can be estimated from resistance measurements of the graphene Hall bar on $\mathrm{LiNbO}_{3}$ (Figure 2(b)). This is connected in series with a mechanical impedance of $310 \Omega$ at the central frequency $f_{0}, R_{a}=\left(f_{0} C_{s} k^{2}\right)^{-1}$, where $C_{s}$ is the finger pair capacitance and $k^{2}$ is the electromechanical coupling constant. This large relative value of $R_{g}$ causes a variation of the voltage along the fingers, which is expected to reduce the transducer efficiency ${ }^{9}$ and can be estimated from the ratio $\vartheta=-10 \log _{10}\left(1+R_{g} / R_{a}\right) \sim-17.2 \mathrm{~dB}$, which quantifies the part of the input energy which dissipates into acoustic radiation. ${ }^{9}$ This value is in close agreement with the difference $(-16.9 \mathrm{~dB})$ between the calculated maximum transmission based on a coupling-of-modes (COM) model for IDTs made of very thin metal electrodes, shown in Figure 4(b), and the measured transmission for as-deposited graphene IDTs in Figure 3(a).

The transmission of the full IDT, calculated using software based on the COM model, also gave good agreement between theory and experiment for the frequency-dependent IDT response, assuming massless metal electrodes, and neglecting reflections. ${ }^{8,13}$ We used a geometry identical to the experiments, except 14 finger pairs were chosen, since this gave results that more closely matched the minima positions in the transmission. Otherwise, the expected transmission was found to be $\sim 1 \mathrm{~dB}$ larger. This small discrepancy with experiment could be explained by a result of mechanical damage, although this is hard to determine owing to the lack of optical contrast between graphene and the substrate. We have also fabricated a pair of IDTs with $\mathrm{Cr} / \mathrm{Au}$ electrodes $(5 \mathrm{~nm} / 50 \mathrm{~nm})$ using the same geometry as shown in Figure 1(b), allowing us to compare graphene to this metallic electrode system (Figure 4(b)). This device gave very close agreement (within $\sim 1 \mathrm{~dB}$ ) to the maximum transmission found from theory. This good agreement allows us to conclude that any effect from diffraction/attenuation of the SAW in lithium niobate (which were not included in the model) is essentially unimportant for our particular IDT geometry. The reason is the strong anisotropy of the substrate, which induces beam steering. ${ }^{12}$ We assume that the background cross-talk level is determined by the close positioning of the IDTs and the geometry of bond wires and sample shielding, as it was found to vary between devices, but the maximum transmission at the IDT centre frequency was found to be identical from one measurement to another.

The finite resistance of graphene limits its application to relatively low frequencies, but at the same time allows us to suggest a type of SAW sensor that cannot be implemented using conventional metal electrodes. In this case, the IDT response is related to the modification of the properties of the IDT itself, rather than a modification to the acoustic path, as is common with metal-electrode-based SAW sensors on lithium niobate as was previously realised. ${ }^{14}$ In the inset of Figure 4(b), the transmission of another device with, and without, an S1813 resist layer is shown (measured at room temperature). A difference of $\sim 1 \mathrm{MHz}$ in the centre frequency was measured after removal of the mass loading caused by the $\sim 1.3-\mu$ m-thick resist. Both these effects, and that of graphene doping, indicate the potential application of graphene IDTs for sensors, in which the electrical properties 
of the graphene IDT are changed (either by mass loading or by doping). We note that such sensors would allow a dramatic reduction in size compared with conventional sensor designs where the operating mechanism is the detection of changes to the properties of the delay line formed from a pair of transducers, including the substrate between them. ${ }^{1,15}$

The effect of temperature on graphene IDT transmission is related to the substrate contraction rather than the change of graphene resistance as a function of temperature. Another way to change resistance without influence on the substrate is to apply perpendicular magnetic field. We explored this at low temperature $2 \mathrm{~K}$ in order to investigate possible contributions from quantum effects to the SAW transmission. Figure 4(c) shows the transmission at $0 \mathrm{~T}$ and $6 \mathrm{~T}$ perpendicular magnetic fields. The effect of magnetic field is expected to come into play from several sources such as magnetoresistance, kinetic inductance and quantum capacitance. ${ }^{16-19}$ However, the last two contributions to the total impedance can be neglected up to very high frequencies. ${ }^{17,19}$ In total the magnetic field has little effect, with only a $0.3 \mathrm{~dB}$ reduction in transmitted signal at $164 \mathrm{MHz}$. This can be directly attributed to magnetoresistance alone as found from the measurements of the graphene Hall bar (Figure 2(b)), the resistivity is modulated by $10 \%$ between $0 \mathrm{~T}$ and $6 \mathrm{~T}$. We believe that non-uniformity in the doping smears out the effect of Shubnikov-de Haas oscillations, which should cause a modulation of the transmission of the order of $\sim 0.5 \mathrm{~dB}$.

Another way to improve the impedance matching would be to increase the total capacitance of the IDT. We note, however, that the number of finger pairs should be increased to achieve this aim rather than the length of individual fingers, since the resistance of fingers increases proportionally to their length. An increase to the central frequency, by decreasing the area of the transducers, could also improve impedance matching, although the voltage along the finger would then obtain a phase shift, which could deteriorate the transducer characteristics. ${ }^{9}$

In conclusion, we have demonstrated the feasibility of forming graphene IDTs at a frequency of $160 \mathrm{MHz}$, and their sensitivity to doping. The effect of cryogenic temperatures on transmission was also studied, and it was shown that the temperature coefficients above $100 \mathrm{~K}$ closely resemble those of metal IDTs formed on $\mathrm{LiNbO}_{3}$. We have also investigated the effect of magnetic field on graphene IDTs performance and found that there is no significant contribution from quantum effects to the SAW transmission.

We acknowledge funding from EPSRC, ERC grants NOTES and TOSCA, the National Physical Laboratory, and support from the Royal Society and the Wolfson Foundation. We are grateful to Roland Clarke and Dr Cinzia Casiraghi for useful discussions, and Dr Oscar Céspedes for use of the Raman spectrometer.

${ }^{1}$ C. K. Campbell, Proc. IEEE 77, 1453 (1989).

${ }^{2}$ M. M. de Lima, Jr., W. Seidel, H. Kostial, and P. V. Santos, J. Appl. Phys. 96, 3494 (2004).

${ }^{3}$ K.-Y. Wong, W. Tang, K. M. Lau, and K. J. Chen, Appl. Phys. Lett. 90, 213506 (2007).

${ }^{4}$ A. K. Geim and K. S. Novoselov, Nature Mater. 6, 183 (2007).

${ }^{5}$ F. Tuinstra and J. Koenig, J. Chem. Phys. 53, 1126 (1970).

${ }^{6}$ A. C. Ferrari, J. C. Meyer, V. Scardaci, C. Casiraghi, M. Lazzeri, F. Mauri, S. Piscanec, D. Jiang, K. S. Novoselov, S. Roth, and A. K. Geim, Phys. Rev. Lett. 97, 187401 (2006).

${ }^{7}$ V. Miseikis, J. E. Cunningham, K. Saeed, R. O’Rorke, and A. G. Davies, Appl. Phys. Lett. 100, 133105 (2012).

${ }^{8} \mathrm{~K}$. Hashimoto, Surface Acoustic Wave Devices in Telecommunications: Modelling and Simulation (Springer, Berlin, Germany, 2000).

${ }^{9}$ K. M. Lakin, IEEE Trans. Microwave Theory Tech. 22, 418 (1974).

${ }^{10}$ S. Bae, H. Kim, Y. Lee, X. Xu, J. Park, Y. Zheng, J. Balakrishnan, T. Lei, H. R. Kim, Y. I. Song, Y. Kim, K. S. Kim, B. Özyilmaz, J. Ahn, B. H. Hong, and S. Iijima, Nat. Nanotechnol. 5, 574 (2010).

${ }^{11}$ F. Schedin, A. K. Geim, S. V. Morozov, E. W. Hill, P. Blake, M. I. Katsnelson, and K. S. Novoselov, Nature Mater. 6, 652 (2007).

${ }^{12}$ C. K. Campbell, Surface Acoustic Wave Devices for Mobile and Wireless Communications (Academic Press Inc., San Diego, 1998), Chaps. 4 and 6.

${ }^{13} \mathrm{~K}$. Hashimoto, COM software Version 1.0. The executable file can be found on the author's website (http://www.te.chiba-u.jp/ ken/).

${ }^{14} \mathrm{Z}$. Zhang, D. Zhu, and Z. Huang, in Proceedings of the 6th International Conference on Solid-State and Integrated-Circuit Technology (2001), Vol. 2, p. 781.

${ }^{15}$ E. F. Whitehead, E. M. Chick, L. Bandhu, L. M. Lawton, and G. R. Nash, Appl. Phys. Lett. 103, 063110 (2013).

${ }^{16}$ P. J. Burke, I. B. Spielman, J. P. Eisenstein, L. N. Pfeiffer, and K. W. West, Appl. Phys. Lett. 76, 745 (2000).

${ }^{17}$ H. S. Skulason, H. V. Nguyen, A. Guermoune, V. Sridharan, M. Siaj, C. Caloz, and T. Szkopek, Appl. Phys. Lett. 99, 153504 (2011).

${ }^{18}$ S. Luryi, Appl. Phys. Lett. 52, 501 (1988).

${ }^{19}$ L. A. Ponomarenko, R. Yang, R. V. Gorbachev, P. Blake, M. I. Katsnelson, K. S. Novoselov, and A. K. Geim, Phys. Rev. Lett. 105, 136801 (2010). 